Revue Française de Civilisation Britannique

\title{
La monnaie d'une Écosse indépendante, enjeu du référendum d'autodétermination de 2014
}

The Currency of an Independent Scotland: a Key Issue in the 2014 Referendum

\section{Edwige Camp-Pietrain}

\section{(2) OpenEdition}

Journals

Édition électronique

URL : http://journals.openedition.org/rfcb/430

DOI : $10.4000 / \mathrm{rfcb} .430$

ISSN : 2429-4373

Éditeur

CRECIB - Centre de recherche et d'études en civilisation britannique

Référence électronique

Edwige Camp-Pietrain, « La monnaie d'une Écosse indépendante, enjeu du référendum

d'autodétermination de 2014 », Revue Française de Civilisation Britannique [En ligne], XX-2 | 2015, mis en ligne le 23 juillet 2015, consulté le 21 avril 2019. URL : http://journals.openedition.org/rfcb/430 ; DOI : $10.4000 /$ rfcb.430

Ce document a été généré automatiquement le 21 avril 2019

\section{(†)

Revue française de civilisation britannique est mis à disposition selon les termes de la licence Creative Commons Attribution - Pas d'Utilisation Commerciale - Pas de Modification 4.0 International. 


\title{
La monnaie d'une Écosse indépendante, enjeu du référendum d'autodétermination de 2014
}

\author{
The Currency of an Independent Scotland: a Key Issue in the 2014 Referendum
}

\author{
Edwige Camp-Pietrain
}

1 Le Scottish National Party (SNP) vise, quasiment depuis sa création en 1934, à restaurer par la voie des urnes la souveraineté de l'Écosse, perdue lors du traité d'Union avec l'Angleterre de 1707. Parvenu à la tête du Gouvernement écossais chargé de gérer les affaires internes dans le cadre de la dévolution du pouvoir, il s'est trouvé en mesure d'organiser un référendum d'autodétermination en 2014. Cependant, alors qu'il entendait instaurer un État, il prônait le maintien de nombreux symboles britanniques de la souveraineté, parmi lesquels la livre sterling et l'union monétaire. Il mettait ainsi en avant la continuité du partenariat entre deux États désormais égaux, afin de convaincre une majorité d'électeurs, au-delà des $30 \%$ prêts à voter pour l'indépendance selon la plupart des sondages jusqu'à la fin de l'année 2013.

Le Gouvernement britannique a accepté de conférer une assise légale à ce référendum, tandis que les partis qui le composaient (Conservateurs et Libéraux-démocrates) lançaient, avec les Travaillistes, une campagne unitaire (Better Together) appelant les électeurs à rejeter l'indépendance. Ils n'ont cessé de dénoncer les risques de cette séparation. La monnaie, qui figurait parmi ces derniers, s'est transformée en question centrale en 2014, lorsque les unionistes ont affirmé que le projet du SNP n'était pas réaliste. Ils voulaient le pousser à opter pour une autre solution (dite "plan B»), notamment une nouvelle devise, plébiscitée par certains alliés du SNP au sein de Yes Scotland, tels les Verts ou le Scottish Socialist Party (SSP).

3 Ce sont les unionistes qui ont axé la campagne sur la monnaie, car ils souhaitaient décrédibiliser l'ensemble du programme nationaliste, fondé sur l'économie. Derrière la monnaie, c'est en effet la nature de l'Écosse indépendante qui se profilait, et, surtout, la faculté de mener des politiques économiques et sociales distinctes. Les électeurs écossais 
ne s'y sont pas trompés. Alors qu'ils se prenaient de passion pour ce débat, la monnaie est devenue un de leurs sujets de conversation courante, même dans ses aspects les plus techniques ${ }^{1}$. Si un nombre croissant d'individus s'est laissé tenter par l'indépendance, ce sont finalement les craintes qui l'ont emporté, $55,3 \%$ des votants repoussant la mise en place d'un État écossais le 18 septembre 2014.

4 Après avoir présenté la position du SNP en la replaçant parmi les options monétaires envisageables dans une Écosse indépendante, nous analyserons l'articulation entre monnaie et souveraineté pendant la campagne, avant d'aborder les effets de cette question sur le résultat du référendum. Il s'agira de montrer comment les deux camps se sont emparés de la monnaie pour façonner l'image d'un État écossais, dans une opposition simplificatrice ${ }^{2}$.

\section{Le SNP face aux choix monétaires d'une Écosse indépendante}

5 Si en 2014, le SNP défendait un projet de continuité, il avait, au cours de son histoire, proposé les quatre options monétaires applicables dans une Écosse indépendante, tout en s'inspirant également d'exemples étrangers. Or chacune de ces orientations permettait de définir l'État qui devait voir le jour.

6 La première option consistait à créer une monnaie. En effet, en endossant l'article 16 du traité d'Union avec l'Angleterre de 1707, les parlementaires écossais avaient opté pour la livre sterling, retirant progressivement leurs pièces et leurs billets (apparus respectivement aux $\mathrm{XII}^{\mathrm{e}}$ et $\mathrm{XVII}{ }^{\mathrm{e}}$ siècles). Les livres «écossaises » restant en circulation étaient dépourvues de cours légal propre ${ }^{3}$. De ce fait, l'indépendance, qui abrogerait ce traité, devrait se traduire par une nouvelle devise. Cette solution, qui témoignerait de la souveraineté retrouvée, était prônée par le SNP jusqu'aux années quatre-vingt-dix. Mais comme elle semblait s'imposer, la réflexion n'avait pas été poursuivie. Les documents de référence publiés par le parti et ses dirigeants (depuis la Déclaration de 1946) n'évoquaient que très brièvement cette question, essentiellement pour souligner la possibilité de s'affranchir de politiques économiques conçues pour le sud-est de l'Angleterre4.

7 La deuxième option se dessina avec la perspective d'une monnaie unique européenne dans le traité de Maastricht signé en 1992 par le Gouvernement de John Major. Le SNP, longtemps réticent à l'égard de la construction européenne (il avait appelé à voter «non» lors du référendum de 1975) avait opéré un revirement en 1988 en promettant l'adhésion à la CEE d'une Écosse indépendante. Ses dirigeants signalaient ainsi que leur nation ne serait pas isolée car elle rejoindrait un grand ensemble. La volonté d'intégrer le mécanisme de change européen, puis la monnaie unique, leur permettait aussi de démontrer les spécificités de l'Écosse alors que l'euroscepticisme gagnait du terrain dans la classe politique anglaise ${ }^{5}$. Le SNP s'entourait toutefois de précautions, la décision finale devant résulter de l'examen de la conjoncture économique écossaise et de l'organisation d'un référendum. Il a maintenu ce projet après 2003, alors que Gordon Brown, Chancelier de l'Échiquier du Gouvernement de Tony Blair, venait de conclure que les conditions n'étaient pas réunies pour le Royaume-Uni ${ }^{6}$. Ainsi, l'indépendance apparaissait comme un moyen pour l'Écosse de faire valoir son engagement européen, qui contrastait avec le 
repli de l'Angleterre. Mais en 2010, en raison de la crise des dettes souveraines dans la zone euro, les nationalistes ont renoncé à la monnaie unique ${ }^{7}$.

8 La troisième option est le fruit de réflexions entamées lorsque le SNP a emporté une majorité absolue de sièges au Parlement écossais (2011), ce qui lui conférait la légitimité politique nécessaire à la convocation d'un référendum d'autodétermination. Le Gouvernement écossais, au pouvoir depuis 2007, s'était déjà entouré d'un conseil économique, sous la houlette de Crawford Beveridge. À partir de ce dernier, il a constitué le groupe de travail de la commission fiscale, composé notamment de deux lauréats du Prix Nobel (James Mirrlees et Joseph Stiglitz). Ce groupe a recommandé le maintien de la livre sterling dans une Écosse indépendante, ainsi que la négociation d'une union monétaire avec le reste du Royaume-Uni. Dans cette optique, l'indépendance devait permettre à l'Écosse de déterminer ses propres politiques économiques, au cœur du programme du SNP, avec une garantie de stabilité financière. La Banque d'Angleterre, banque centrale, accorderait un soutien en cas de difficultés d'un établissement financier: elle demeurerait prêteur de dernier ressort ${ }^{8}$. Cette hypothèse n'était pas anecdotique car l'Écosse avait déjà connu cette situation à la fin de 2008, deux de ses fleurons (Royal Bank of Scotland, RBS et Bank of Scotland, BoS), à court de liquidités, ayant été sauvés par les prises de participations majoritaires du Gouvernement britannique9.

Une quatrième solution, qui a émergé au cours de la campagne référendaire, ne pouvait être écartée par le SNP. En effet, le 13 février 2014, George Osborne, Conservateur, Chancelier de l'Échiquier, a catégoriquement affirmé que le Gouvernement britannique refuserait toute union monétaire avec une Écosse indépendante, en raison des risques pour le contribuable du reste du Royaume-Uni ${ }^{10}$. Cette analyse a aussitôt été endossée par ses alliés au sein de Better Together, par les voix de Danny Alexander, ministre (libéraldémocrate) au Trésor et d'Ed Balls, porte-parole pour les questions financières au Parti travailliste, ainsi que par Carwyn Jones, First Minister (travailliste) du pays de Galles. Dès lors, si le Gouvernement écossais devait persister dans son intention de conserver la livre, il devrait opérer sans union monétaire, ce qui l'obligerait notamment à prévoir des solutions destinées à pallier toute crise financière en l'absence de prêteur de dernier ressort. L'indépendance était donc assimilée à un saut dans l'inconnu. En août 2014, cette question a occupé une grande partie des débats télévisés entre Alex Salmond, leader du SNP et First Minister, et Alistair Darling, leader (travailliste) de Better Together. Face à l'unanimité des unionistes, même si le premier a continué à afficher sa résolution à obtenir une union monétaire dans le cadre de négociations globales avec le Gouvernement britannique, il ne pouvait plus ignorer cette «sterlingisation» qui s'imposerait par défaut. Or, le terme, forgé par analogie avec la «dollarisation» de Panama, était aux antipodes de la gestion rigoureuse et autonome prônée par le Gouvernement écossais.

Le SNP ne pouvait compter sur l'unanimité du camp indépendantiste. Nombre de ses alliés plébiscitaient la création d'une monnaie, seul gage d'une véritable indépendance. Il s'agissait notamment de membres du comité consultatif de Yes Scotland, son leader, Dennis Canavan (ancien travailliste), ainsi que Patrick Harvie et Colin Fox (respectivement leaders des Verts et du SSP). S'ajoutaient d'anciens dirigeants du parti (Jim Sillars, qui fut numéro deux de Salmond, Gordon Wilson, prédécesseur de ce dernier, ainsi que Jim Fairlie) et un nouveau mouvement, la Radical Independence Campaign. Tous ces indépendantistes voulaient doter les dirigeants écossais des moyens de réformer l'Écosse en profondeur. Avec leur devise, ils pourraient concevoir leurs propres politiques 
monétaires, mais aussi budgétaires et fiscales, afin de mettre un terme à l'orthodoxie néolibérale ${ }^{11}$. L'indépendance apparaissait donc comme une véritable rupture. Mais ces indépendantistes étaient prêts à mettre en sourdine leurs divergences, estimant que leur priorité, pendant la campagne, était de persuader les Écossais de voter en faveur de l'indépendance ${ }^{12}$.

11 Par ailleurs, si le SNP se plaisait à citer des petits États, notamment en Europe du nord (qualifiée d' «arc de prospérité » jusqu'en 2008), pour conforter la viabilité économique de l'indépendance, il ne pouvait se prévaloir d'un modèle uniforme ${ }^{13}$. Mais il devait constater que chaque État avait effectué des choix monétaires visant à assurer un degré de stabilité avec la monnaie de son principal partenaire commercial, l'indépendance devant s'accompagner de garanties. Ainsi, le Danemark, la Suède et la Norvège avaient leur devise. Les deux premiers, membres de l'UE, ont choisi de ne pas adhérer à l'euro, le premier disposant même d'une dérogation permanente comme le Royaume-Uni. Mais ils ont mis en place des mécanismes d'adossement à la monnaie unique européenne. L'Irlande a préféré adopter celle-ci, dès sa mise en circulation. Cela constituait l'aboutissement d'un processus d'émancipation progressive à l'égard du Royaume-Uni, sur les plans monétaire, mais aussi économique et commercial. Peu après son indépendance, l'Irlande avait opté pour sa monnaie, maintenant une parité fixe avec la livre sterling dans le cadre d'un conseil monétaire (currency board), avant de se doter d'une banque centrale assumant les fonctions de prêteur de dernier ressort (1955) et d'élargir le panier de devises de référence. Or en 2014, le système du conseil monétaire était en vigueur dans des micro-territoires (Hong Kong, îles anglo-normandes, île de Man) que le SNP ne pouvait revendiquer comme des exemples car la taille de l'Écosse indépendante et le poids de son secteur financier seraient nettement supérieurs. Cependant, en juillet 2013, Alex Salmond avait entretenu une certaine ambiguïté en se rendant sur l'île de Man pour mettre en évidence l'existence de diverses options monétaires permettant d'assurer la prospérité et la stabilité, dûment reconnue par les agences de notation.

12 Ces options monétaires qui orientaient l'image et, partant, le degré de souveraineté de l'Écosse indépendante, sont devenues des thèmes majeurs de la campagne.

\section{L'imbrication entre monnaie et souveraineté pendant la campagne}

13 Les débats autour de la monnaie concernaient la souveraineté de l'Écosse indépendante, s'agissant autant de son statut à l'égard du reste du Royaume-Uni que de sa capacité à mener des politiques autonomes.

14 S'agissant du statut de l'Écosse, le Gouvernement écossais, appuyé par sa commission fiscale, justifiait l'accession à l'indépendance dans la continuité et ce, dans l'intérêt des gouvernements et des acteurs économiques, en Écosse et dans le reste du Royaume-Uni ${ }^{14}$. L'Écosse conserverait des institutions qu'elle avait contribué à instaurer, lesquelles se mettraient au service de deux États. Ainsi, la Banque d'Angleterre (fondée en 1694 par un Écossais, William Paterson) tiendrait désormais compte de la conjoncture en Écosse lors de la fixation de ses taux directeurs. Elle soutiendrait les établissements financiers écossais le cas échéant; ceux-ci ayant une clientèle majoritairement anglaise, elle tenterait alors de prévenir un risque systémique. D'ailleurs, le Gouvernement écossais 
avait entamé des discussions techniques avec la Banque d'Angleterre pour préparer une éventuelle transition. Qui plus est, les relations économiques entre Écosse et reste du Royaume-Uni étaient étroites. L'Écosse réalisait la plupart de ses échanges de biens (deux-tiers) et de ses mouvements de personnes (50\% des flux migratoires) avec l'Angleterre, tandis que les entreprises anglaises en Écosse étaient à l'origine de plus de $20 \%$ des emplois locaux. L'Écosse constituerait le deuxième partenaire commercial du reste du Royaume-Uni. L'adoption de monnaies différentes alourdirait le coût des transactions, et ce, même pour les Anglais (à hauteur de 500 millions de livres). En outre, l'Écosse et l'Angleterre connaissant des cycles économiques globalement convergents, l'union monétaire serait cohérente, même pour cette dernière qui tirerait profit des atouts écossais (notamment l'exportation d'hydrocarbures) pour stabiliser sa balance des paiements. La souveraineté retrouvée de l'Écosse serait donc profitable à l'ensemble du Royaume-Uni.

15 À l'inverse, dès le mois d'avril 2013, dans le deuxième opus de la série 'Scotland Analysis', le Gouvernement britannique a dénoncé ce raisonnement ${ }^{15}$. Selon les unionistes, seul le reste du Royaume-Uni succéderait au Royaume-Uni existant. Le Gouvernement écossais, à l'initiative de la rupture, ne recouvrerait qu'une souveraineté théorique car il devrait affronter de multiples contraintes. Les opérateurs financiers devraient supporter des frais supplémentaires, liés à l'obligation de mise en place d'une autorité écossaise de régulation des marchés. Ils encourraient des risques. En effet, la Banque d'Angleterre cesserait d'être la banque centrale d'un État devenu indépendant, car elle ne pouvait être responsable qu'auprès d'un seul gouvernement. Elle ne serait pas en mesure de tenir compte des intérêts de l'Écosse car son conseil monétaire était composé d'experts recrutés pour leurs compétences et non en fonction de leur origine géographique. En cas de crise, l'Écosse devrait assurer le sauvetage de ses établissements. Or les actifs des banques écossaises représentaient $492 \%$ du PIB britannique, mais $1254 \%$ du seul PIB écossais. L'intervention des autorités britanniques en 2008 ne pourrait être aisément reproduite par les autorités écossaises indépendantes. L'Irlande, dont le secteur bancaire représentait neuf fois le PIB, avait dû s'endetter massivement pour le sauver. Si la Banque d'Angleterre consentait à apporter son aide, elle prescrirait des contraintes drastiques.

Par ailleurs, l'Écosse devrait affirmer sa crédibilité auprès des marchés internationaux. Selon les nationalistes, ses ressources économiques et son histoire au sein du RoyaumeUni permettraient au Gouvernement écossais d'emprunter dans des conditions aussi avantageuses que son homologue britannique; la note AAA semblait acquise. Mais les unionistes rétorquaient qu'en l'absence de bilan propre, les obligations émises par l'État écossais se verraient infliger un surcoût, de 0,72 à 1,65 point de pourcentage ${ }^{16}$, qui se répercuterait aussitôt sur les taux d'intérêts pratiqués par les établissements financiers écossais auprès de leurs clients. Pis, les marchés avaient démontré qu'ils pouvaient mettre en échec tout arrangement dépourvu de fondation solide, entre deux partenaires inégaux. Ainsi, en 1993, l'union monétaire prévue lors de la partition de la Tchécoslovaquie avait été interrompue dès le deuxième mois sous la pression des spéculateurs.

17 Les dettes contractées par le nouvel État s'additionneraient aux dettes britanniques. Certes, juridiquement, seul le Gouvernement du Royaume-Uni était responsable de ces dernières, comme il l'avait reconnu le 13 janvier 2014, afin de rassurer les investisseurs ${ }^{17}$. Mais les unionistes avaient aussitôt ajouté que le Gouvernement d'une Écosse indépendante ne pourrait raisonnablement s'affranchir du remboursement de la part lui 
revenant sur la base de sa population. Toute renonciation serait assimilée à un défaut, entachant gravement sa réputation, auprès des investisseurs, des agences de notation, mais aussi de l'UE, à laquelle le SNP souhaitait adhérer. À l'origine, le Gouvernement écossais, et ses conseillers, envisageaient une répartition de cette dette, selon une méthode plus favorable à l'Écosse (liée à la contribution historique de celle-ci à l'endettement) que celle prônée par le Gouvernement du Royaume-Uni ${ }^{18}$. Mais, à l'issue de la déclaration de George Osborne, les nationalistes ont laissé entendre que si le reste du Royaume-Uni refusait toute union monétaire, c'est-à-dire tout partage des institutions communes (parmi lesquelles, la monnaie et la Banque d'Angleterre), l'Écosse indépendante ne se sentirait pas liée par son passif. Alex Salmond s'est montré très explicite lors des débats télévisés avec Alistair Darling en août 2014. Ce dernier a toutefois dénoncé le caractère simplificateur de cette vision, comme si l'indépendance pouvait se résumer au démembrement d'un patrimoine sur le modèle d'un divorce entre personnes ; en tout état de cause, la monnaie n'était pas comparable à une collection de CD car elle était fondée sur la confiance des opérateurs à l'égard d'un État ${ }^{19}$.

En ce qui concerne la capacité à mener des politiques autonomes, le Gouvernement écossais entendait pratiquer des politiques de relance en réduisant l'impôt sur les sociétés (en-deçà du taux pratiqué par le reste du Royaume-Uni) et en empruntant pour financer des hausses de dépenses publiques. Il jugeait ce projet compatible avec une union monétaire car après avoir défini en commun les grands équilibres budgétaires, les Gouvernements écossais et britannique seraient libres de sélectionner les moyens pour les atteindre.

Dans un premier temps, les autorités britanniques ont émis des doutes. Ainsi, en avril 2013, le Gouvernement britannique avait pris l'exemple de la zone euro pour démontrer qu'une union monétaire devait reposer sur une union politique. Dans une union monétaire entre l'Écosse et le reste du Royaume-Uni, c'est l'Angleterre qui dicterait ses choix, notamment l'objectif en matière de taux d'inflation, ainsi que des politiques économiques et sociales plus axées sur la rigueur. Le 29 janvier 2014, Mark Carney, gouverneur de la Banque d'Angleterre, tout en se défendant de prendre parti, avait souligné que toute union monétaire impliquait un alignement des politiques budgétaires et, partant, une érosion de la souveraineté nationale ${ }^{20}$.

En février 2014, le discours d'Osborne a marqué un tournant. Il s'est appuyé sur les conseils du secrétaire permanent du Trésor, Nicholas MacPherson, sachant que l'avis émis par cette institution en 2003 au sujet de l'euro avait prouvé sa pertinence depuis lors. Le Chancelier a indiqué qu'au terme d'une analyse approfondie, le maintien d'une union monétaire paraissait inconcevable ${ }^{21}$. Le contribuable du reste du Royaume-Uni ne saurait être sollicité pour aider des Écossais ayant opté pour l'indépendance ${ }^{22}$. Or non seulement l'Écosse se livrerait à un dumping fiscal susceptible de détruire des emplois en Angleterre mais, qui plus est, elle serait vulnérable aux chocs extérieurs (étant donné sa dépendance à l'égard du pétrole) et fragilisée par la nécessité de protéger son secteur financier. Le Gouvernement britannique rappelait que l'Irlande avait vu son endettement public passer de $25 \%$ de son PIB en 2007 à $100 \%$ pour sauver ses banques. Encore l'Irlande appartenait-elle à la zone euro. Mais la situation écossaise serait plutôt à rapprocher de celles d'États comme le Panama, subordonnés à un voisin plus puissant. Sa «sterlingisation» l'obligerait à constituer d'énormes réserves de change (estimées par Mark Carney à $25 \%$ du PIB) pour faire face aux aléas. Cela exigerait des ajustements budgétaires et des politiques d'austéritée ${ }^{23}$. 
21 Les Travaillistes ont corroboré le raisonnement du Gouvernement britannique. Alistair Darling s'est positionné en tant qu'ancien Chancelier de l'Échiquier du Gouvernement de Gordon Brown, responsable du sauvetage des banques écossaises. Certains de ses collègues qui avaient préféré mener une campagne autonome (United with Labour) ont mis l'accent sur les effets concrets d'une rupture, sur les remboursements de prêts hypothécaires (étant donné la pratique répandue des taux d'intérêts variables) ou sur le montant des retraites (qui ne pourrait être garanti contrairement aux promesses du SNP). Gordon Brown lui-même, initialement circonspect à l'égard des propos d'Osborne qui opposaient abruptement l'Écosse et le reste du Royaume-Uni, n'a pas hésité à évoquer la perspective d'un statut « néo-colonial » en août 2014, à l'approche du scrutin ${ }^{24}$.

En dépit de ces arguments, Salmond a maintenu sa position lors des deux débats télévisés face au questionnement incisif de Darling. Il affirmait défendre les droits et les intérêts des Écossais en sélectionnant la «meilleure » option. Il n'a pas fléchi car il craignait de heurter les électeurs indécis, qu'il devait convaincre pour emporter ce référendum. Pourtant, c'est l'ensemble de la politique économique du Gouvernement écossais qui perdait sa crédibilité. Tout au plus, entre les deux débats, lorsque cette question a semblé devenir centrale, son conseiller Crawford Beveridge a-t-il laissé entrevoir la perspective d'une utilisation de la livre sans union monétaire, en minorant les conséquences sur les politiques budgétaires ${ }^{25}$. En effet, selon lui, non seulement le Gouvernement britannique surestimait le poids des actifs bancaires en Écosse, mais, qui plus est, il passait volontairement sous silence la faculté de faire appel à des banques centrales extérieures ou aux marchés monétaires en cas de crise de liquidités, au lieu de constituer des réserves de change au préalable.

Cependant, le Chancelier avait employé des termes laissant penser que cette « sterlingisation " n'était même pas envisageable car les Écossais ne pourraient plus faire usage de la livre sterling s'ils quittaient le Royaume-Uni. L'objectif affiché était de contraindre le SNP à opter pour une autre monnaie, même si les unionistes entendaient en réalité multiplier les critiques visant à prouver l'incompétence du Gouvernement écossais. La commission des Affaires écossaises à la Chambre des Communes, dominée par les Travaillistes, a aussitôt repris cette position ${ }^{26}$, pourtant jugée choquante par de nombreux observateurs écossais ${ }^{27}$.

D'une part, la solution la plus évidente semblait être la création d'une monnaie. De nombreux indépendantistes la plébiscitaient afin de permettre à un État écossais de mener ses propres politiques, monétaires mais aussi budgétaires, sans avoir de compte à rendre à une autorité extérieure. Il pourrait avoir recours à la dévaluation afin de financer des politiques sociales ambitieuses. Par ailleurs, à la différence du SNP, ces indépendantistes souhaitaient augmenter les impôts sur les profits des entreprises et sur les hauts revenus, tout en mettant fin à l'autonomie des autorités monétaires à l'égard du pouvoir politique ${ }^{28}$.

Mais le SNP n'était pas prêt à promouvoir cette option, la plus coûteuse et la plus risquée sur le court terme car il faudrait concevoir un système monétaire, et, surtout, susciter la confiance des opérateurs. Or le SNP voulait éviter les moqueries relatives à la valeur de la livre écossaise avant l'Union (laquelle avait connu de nombreuses dévaluations par rapport à la livre sterling ${ }^{29}$ ) ou à la dénomination de la nouvelle devise (la presse britannique ayant exhibé les divers noms employés au Moyen-Âge). Il voulait également échapper à la personnalisation de la question, d'autant qu'entre les deux débats télévisés, Jim Murphy (député travailliste à la Chambre des Communes) a présenté, au nom de Better 
Together, une affiche simulant une pièce écossaise à l'effigie d'Alex Salmond. Or le First Minister, responsable politique écossais le plus populaire, s'attirait également de solides inimitiés ${ }^{30}$. Sur le fond, les unionistes soutenaient que cette monnaie écossaise aurait tendance à subir des réévaluations dues à l'afflux de devises provenant des recettes pétrolières, ce qui porterait préjudice aux exportations des autres secteurs. Certes, le SNP rappelait que ce type de difficultés avait été surmonté par la Norvège : comme elle, il entendait mettre en place un fonds d'épargne. Mais les unionistes ont émis des doutes quant à la constitution de celui-ci dans une phase de déficit budgétaire.

D'autre part, une monnaie était disponible, l'euro. Or le SNP prônait l'adhésion d'une Écosse indépendante à l'UE, selon une procédure rapide et simplifiée. De ce fait, les unionistes n'ont pas manqué de souligner que celle-ci serait retardée par la volonté de conserver la livre sterling alors que tout nouveau membre devait prendre l'engagement de rejoindre la monnaie unique. Cela impliquait la création d'une monnaie nationale, l'intégration au mécanisme de change européen et le respect de critères stricts (déficit inférieur à $3 \%$ du PIB, dette en-deçà de $60 \%$ ). Ceux-ci semblaient hors de portée en 2016, année de l'accession à l'indépendance, les estimations du SNP avoisinant respectivement $6 \%$ et $70 \%$. Des ajustements budgétaires sévères étaient à prévoir. Ainsi, les unionistes mettaient une fois encore l'accent sur l'impossibilité d'avoir recours à des politiques de relance, face aux conditions imposées par la Banque Centrale Européenne. Ils laissaient également planer le doute sur un élément central du programme du SNP, l'appartenance à l'UE et, notamment, à son marché unique, destinée à prouver qu'elle ne serait pas isolée 31 .

Cette articulation étroite entre monnaie et souveraineté, loin d'être confinée aux discussions entre experts, a passionné la société civile.

\section{La monnaie, préoccupation majeure de la société civile}

La déclaration de George Osborne rejetant toute union monétaire a engendré deux types d'effets potentiellement contradictoires. Caractéristiques de cette campagne référendaire, ils ont été exploités par les deux camps jusqu'au jour du scrutin et ont eu un impact sur le résultat.

D'une part, selon les nationalistes, ce refus reflétait la volonté de l'élite de «Londres » de dicter aux Écossais ce que devait être leur conduite. Ils décriaient également la méthode, brutale et calculée, le Chancelier ayant fait un passage éclair en Écosse dans cette intention en février 2014. De plus, cette attitude, autrefois associée aux Conservateurs (dont Osborne et Cameron constituaient encore, en 2014, des figures emblématiques), se retrouvait désormais chez les Travaillistes qui s'étaient empressés de soutenir les arguments de leurs opposants traditionnels. Même la fonction publique britannique était politisée, comme en témoignait la diffusion par le Chancelier de l'avis de son secrétaire permanent. Aucune réforme ne semblant envisageable, la seule issue était l'accession à l'indépendance.

30 Face à cet " establishment politique », Salmond s'est érigé en représentant authentique des intérêts écossais, notamment au cours des deux débats télévisés. Lors du premier, il a récusé tout plan B parce qu'il se devait de défendre la meilleure solution pour son peuple. Il a tenté de renvoyer Darling à son bilan en tant que Chancelier de l'Échiquier, insinuant 
qu'il était responsable de la crise financière. Lors du second débat, le First Minister a expliqué qu'un vote en faveur de l'indépendance lui permettrait de se présenter devant le Gouvernement britannique pourvu d'un mandat du peuple écossais, afin de négocier une union monétaire, mandat qui ne pourrait alors être ignoré. Il a questionné Darling sur la position qu'il adopterait en tant qu'Écossais, député à la Chambre des Communes. Dans tous les cas, personne ne pouvait empêcher les Écossais de continuer à faire usage de leur monnaie. En retour, Darling, comme ses alliés unionistes, ont dénoncé l'arrogance de Salmond, cantonné dans un raisonnement contredit par des analyses économiques minutieuses. Le First Minister semblait vouloir avoir raison contre tous ses adversaires politiques et contre la plupart des experts.

D'autre part, l'intervention du Chancelier a libéré la parole des dirigeants d'entreprises qui, selon les unionistes, hésitaient à s'exprimer en raison des pressions exercées par le Gouvernement écossais. Ainsi, au début de l'année 2014, des banques (RBS, groupe Lloyds) et des sociétés d'assurance-vie (Standard Life) ont fait état d'incertitudes pour leur activité. De même, la Confederation of British Industry (CBI), principale organisation patronale, s'est appuyée sur les déclarations du ministre pour justifier son scepticisme à l'égard de l'indépendance ${ }^{32}$. Mais les nationalistes ont rétorqué que l'incertitude était imputable au seul Gouvernement britannique qui s'opposait à leur projet d'union monétaire visant à garantir la continuité. De plus, Ross McEwan, directeur général de RBS, avait précisé que son établissement, présent dans plus de 30 pays, était habitué à composer dans des environnements monétaires et réglementaires fort divers.

Ces prises de position ont connu un deuxième temps fort à l'approche du référendum. Les deux camps ont exhibé des listes de plus de 100 noms de chefs d'entreprises pour et contre l'indépendance. Or parmi les raisons citées par les plus réticents, l'incertitude liée à la monnaie figurait en bonne place; de même, Keith Cochrane, PDG du groupe d'ingénierie pétrolière Weir, jugeait cette question essentielle ${ }^{33}$. À l'inverse, les chefs d'entreprises en faveur de l'indépendance y voyaient une opportunité, minorant les risques. Ainsi, la monnaie était absente de leur argumentaire.

Les controverses ont surtout pris de l'ampleur après la publication, par le Sunday Times du 7 septembre, d'un sondage commandé à YouGov, selon lequel $51 \%$ des Écossais étaient prêts à voter pour l'indépendance. En réaction, le 10, les trois leaders des partis britanniques se sont rendus en Écosse, réitérant leur engagement à poursuivre le transfert de pouvoirs au Parlement écossais en cas de rejet de l'indépendance. Le même jour, les dirigeants de RBS, Lloyds et de Standard Life, ainsi que ceux de TSB, Clydesdale et de Tesco Bank, ont indiqué qu'ils envisageaient la création d'entités juridiques en Angleterre, afin de transférer tout ou partie de leur siège social là où se trouvaient la majorité de leurs clients. Ils souhaitaient préserver leur crédibilité (reposant sur la confiance des particuliers et des marchés) et leur assise légale, notamment en raison des incertitudes liées au prêteur de dernier ressort. De plus, les dirigeants de deux géants de la grande distribution, John Lewis et Asda, ont estimé que les prix seraient plus élevés dans une Écosse indépendante ${ }^{34}$.

En réaction, Salmond a crié au complot, notamment pour RBS (dont l'État détenait toujours plus de $80 \%$ ), exigeant une enquête. Il brandissait une nouvelle preuve de la puissance de l'establishment britannique, auquel il associait désormais la $B B C$, alors que le Gouvernement écossais s'était jusque-là gardé de cautionner les accusations de partialité proférées par les indépendantistes les plus radicaux à l'égard de la chaîne publique. Pourtant, la force de frappe des partis britanniques et de leurs relais dans les 
milieux économiques et financiers comme dans la presse ne laissait pas le SNP impuissant. Alex Salmond et John Swinney, son ministre des Finances, recueillaient les fruits d'un patient travail de discussion avec des chefs d'entreprises, en petits groupes, dans les années 2000. Ainsi, Business for Scotland se targuait de 3000 membres en faveur de l'indépendance, parmi les grandes entreprises comme parmi les PME. En septembre 2014, Salmond s'est prévalu du soutien de patrons d'un gestionnaire de fonds (Martin Gilbert, Aberdeen Asset management) et d'un banquier d'affaires (Angus Grossart, fondateur de Noble \& Grossart) qui dénonçaient l'exagération des mises en garde. De même, Tesco, leader de la grande distribution en Grande-Bretagne, et numéro deux mondial, n'avait pas pronostiqué de hausse des prix. Mais seuls les propos des dirigeants de grandes banques et des autres distributeurs, repris par les médias britanniques, paraissaient audibles. Jim Sillars, amer, a laissé entendre que le Gouvernement écossais saurait se souvenir de ces prises de position, tandis que Salmond, plus fédérateur, se disait prêt à travailler avec tous les acteurs économiques ${ }^{35}$.

35 La dramaturgie était accentuée par le fait que ces déclarations se sont accompagnées d'une chute de la livre sterling, à un étiage jamais atteint depuis le début de l'année 2014. Même si celle-ci a remonté dès le sondage suivant, qui donnait le «non " gagnant, une crise était probable en cas de victoire du « oui ». Mark Carney a conseillé aux banques de faire le plein de billets dans leurs distributeurs automatiques, avant d'annoncer son retour anticipé d'Australie pour la proclamation des résultats. De plus, il affirmait que son organisme assurerait la transition, notamment la garantie des dépôts.

Cette situation rappelait les deux campagnes précédant les référendums sur la dévolution, au cours desquelles les Conservateurs avaient incité des chefs d'entreprises à manifester leurs craintes, notamment au sujet de hausses d'impôts. Or les compétences sur la fiscalité des entreprises des assemblées prévues à l'époque étaient inexistantes (1979) ou très faibles $(1997)^{36}$. Au-delà se profilait surtout la menace de délocalisations et de pertes d'emplois. En 2014, les responsables d'établissements financiers n'envisageaient pas de licenciements, préférant souligner la nécessité de mise en cohérence réglementaire de leurs activités. Mais les dirigeants nationalistes savaient, sans l'admettre publiquement, que le déplacement des sièges sociaux des banques rendrait caduques les principales critiques opposées à une union monétaire. En effet, il n'y aurait plus de grands établissements financiers " écossais », susceptibles de réclamer l'aide d'un prêteur de dernier ressort à une institution sise dans un autre État.

37 En ce qui concerne l'impact de ces controverses sur les comportements électoraux, l'union monétaire était l'option la plus populaire, approuvée par près de $60 \%$ des Écossais entre février et septembre 2014 (sondages ICM) ${ }^{37}$. Cette stabilité en fin de campagne semblait démontrer que les assertions d'Osborne manquaient de crédibilité, nombre d'électeurs y décelant une tentative d'intimidation. Mais cette question était également devenue une source de divisions potentielles entre Écossais et Anglais, puisque l'union monétaire n'était prônée que par un quart de ces derniers (YouGov) ${ }^{38}$.

La dénonciation de l'élite britannique agissant de concert a probablement conforté les intentions de voter « oui » qui sont passées, en moyenne, de $41 \%$ à $43 \%$, en mars, et de $44 \%$ à $46 \%$ à la fin août et qui avoisinaient $48 \%$ à quelques jours du scrutin. Cependant, seuls $7 \%$ des partisans de l'indépendance ont indiqué que leur choix était lié à la monnaie, selon le sondage financé par Lord Ashcroft le jour du scrutin ${ }^{39}$. Le dernier bond de l'opinion est en grande partie imputable à l'accent mis, par le Gouvernement écossais, sur les politiques britanniques d'austérité et leurs effets délétères en Écosse (explosion de 
la pauvreté, menace sur le NHS). C'est d'ailleurs la raison pour laquelle nombre de syndicats, ainsi que la confédération STUC, avaient refusé d'appeler au rejet de l'indépendance ${ }^{40}$. Le STUC reconnaissait que l'union monétaire priverait l'Écosse de moyens d'action autonome, tout en s'insurgeant contre la position fermée d'Osborne ${ }^{41}$.

Dans le même temps, les derniers indécis se sont montrés sensibles aux discours anxiogènes. Ainsi, la monnaie était la principale raison motivant un vote contre l'indépendance, retenue par $57 \%$ des sondés pour l'enquête de Lord Ashcroft. Le Royaume-Uni semblait synonyme de sécurité comme l'avait martelé Better Together pendant deux ans.

\section{Conclusion}

La monnaie s'est indiscutablement imposée dans la campagne référendaire en 2014, avec deux points d'orgue : la déclaration de George Osborne, en février, et les deux débats télévisés entre Alex Salmond et Alistair Darling, en août. Ce sujet, théoriquement technique, réservé aux économistes, est devenu un objet de discussions courantes car il devait affecter la vie quotidienne: salaires, épargne, retraites, allocations, achats de biens. Il était donc étroitement lié à de multiples thèmes de la campagne. Son traitement par les deux camps était emblématique de leur stratégie globale.

41 Les nationalistes, qui voulaient rassurer en garantissant la continuité, selon des critères économiques, se sont trouvés sur la défensive, devant justifier un choix politique, autour d'un élément central de la souveraineté. Même sur le plan économique, le débat était loin d'être tranché. Ainsi, deux anciens dirigeants de Scottish Enterprise, organisme chargé d'attirer les investisseurs en Écosse, avaient des lectures divergentes: Crawford Beveridge, conseiller du Gouvernement, plébiscitait l'union monétaire, tandis que Jack Perry doutait de sa faisabilité. De même, parmi les lauréats du Prix Nobel, Paul Krugman s'opposait aux deux conseillers du Gouvernement écossais.

Les unionistes ont martelé que l'option retenue par le SNP était risquée, avant d'ajouter qu'elle était inapplicable, afin de le contraindre à dévoiler un plan $\mathrm{B}$ qui aurait été encore plus exposé à la critique relative à la capacité d'action autonome de l'État écossais. Néanmoins, en novembre 2014, Dennis Canavan, leader du comité consultatif de Yes Scotland, a estimé que la position du Gouvernement écossais avait contribué à la défaite ${ }^{42}$. De même, Robin McAlpine, directeur de Common Weal, une boîte à idées qui a fait campagne aux côtés de Yes Scotland, a regretté l'absence de contre-attaque plus étayée, sur le terrain économique, face aux menaces des unionistes. Mais ces reproches, émis a posteriori, étaient stimulés par le score élevé des indépendantistes, alors que le SNP avait privilégié la prudence en raison de sondages d'opinion peu favorables.

Au-delà, dans cette campagne, c'est la crédibilité de l'ensemble du Royaume-Uni en tant que place financière mondiale qui était en jeu. Si l'indépendance avait recueilli une majorité de suffrages, les deux camps auraient sans doute dû se résoudre au compromis. Certes, les délocalisations des sièges sociaux des principaux établissements financiers auraient levé nombre d'obstacles. Mais des sources ministérielles avaient laissé entendre que le Gouvernement britannique, obnubilé par la volonté du SNP de débarrasser le sol écossais des armes de dissuasion nucléaire, aurait pu se montrer conciliant sur la question de la monnaie ${ }^{43}$. 
Alex Salmond était à l'origine du positionnement du SNP. Pour l'avenir, Nicola Sturgeon, qui lui a succédé à la tête du parti et du Gouvernement écossais, pourrait envisager une clarification, dans la perspective de deux échéances majeures. D'une part, le SNP compte rassembler les voix émises en faveur de l'indépendance de l'Écosse pour parvenir à devancer les Travaillistes lors des élections à la Chambre des Communes de mai 2015, ce qui serait sans précédent dans ce type de scrutin. Il doit néanmoins tenir compte des controverses suscitées par son programme. D'autre part, si les Conservateurs sont reconduits au plan britannique lors de ce scrutin, David Cameron a promis la convocation d'un référendum sur l'UE avant la fin 2017. La nouvelle First Minister écossaise a laissé entrevoir la possibilité d'un second référendum d'autodétermination, les Écossais ne pouvant accepter une sortie de l'UE à l'initiative des électeurs anglais. Or la déclaration d'Osborne, tout comme le discours prononcé en janvier 2015 par Nicholas MacPherson, secrétaire permanent du Trésor, reconnaissant le caractère unioniste de cette institution ${ }^{44}$, ont fait voler en éclat l'idée d'un partenariat entre égaux, développée par le SNP en 2014. Cette brèche dans l'Union pourrait précipiter l'Écosse vers l'indépendance ${ }^{45}$, d'autant qu'elle s'accompagne d'interrogations, de la part des Conservateurs, sur la place des députés écossais à la Chambre des Communes.

\section{BIBLIOGRAPHIE}

ARMSTRONG, Angus \& EBELL, Monique. Scotland's Currency Options. Londres : National Institute of Economic and Social Research, octobre 2013, 47 p.

ARMSTRONG, Angus \& EBELL, Monique. "Scotland: Currency Options and Public Debt”, National Institute Economic Review, n²27, février 2014, R14-R20.

ARMSTRONG, Angus \& EBELL, Monique. Assets and Liabilities of Scottish Independence. Londres : National Institute of Economic and Social Research, discussion paper $\mathrm{n}^{\circ} 426$, avril 2014, $14 \mathrm{p}$.

ARMSTRONG, Angus \& EBELL, Monique. "Commentary: Monetary Unions and Fiscal Constraints”, National Institute Economic Review, n²28, mai 2014, F4-F11.

ARMSTRONG, Angus \& EBELL, Monique. Is this Plan B?. Londres : National Institute of Economic and Social Research, septembre 2014, 8 p.

ARMSTRONG, Angus \& McCARTHY, David. Scotland's Lender of Last Resort Options. Londres : National Institute of Economic and Social Research, discussion paper n ${ }^{\circ} 434$, août 2014, $37 \mathrm{p}$.

BROWN, Gordon. My Scotland, Our Britain: a Future Worth Sharing. Londres : Simon \& Schuster, 2014, $354 \mathrm{p}$.

CAMP, Edwige. « Les Pressions des milieux économiques et financiers sur le processus de dévolution en Écosse ", Babel, n²17, 2008, pp. 211-236.

CAMP-PIETRAIN, Edwige. L'Écosse et la tentation de l'indépendance. Le référendum d'autodétermination de 2014. Villeneuve d'Ascq : Presses universitaires du Septentrion, avril 2014, 216 p.

CAMP-PIETRAIN, Edwige. L'impossible indépendance écossaise ?. Neuilly : Atlande, octobre 2014, 224 p. 
CARNEY, Mark. The Economics of Currency Unions. Édimbourg : Scottish Council for Development and Industry, janvier 2014, $20 \mathrm{p}$.

COCHRANE, Alan. Alex Salmond: My Part in his Downfall. Londres : Biteback, 2014, 324 p.

CONFEDERATION OF BRITISH INDUSTRY. The Scottish Government's Plans for Independence. Glasgow, $2014,30 \mathrm{p}$.

DUCLOS, Nathalie. L'Écosse en quête d'indépendance? Le référendum de 2014. Paris : Presses de l'université de Paris-Sorbonne, juillet 2014, 299 p.

FISCAL COMMISSION WORKING GROUP. First Report-Macroeconomic Framework. Édimbourg : Scottish Government, février 2013, 198 p.

FISCAL COMMISSION WORKING GROUP. The Economic Foundations of an Independent Scotland. Édimbourg : Scottish Government, août 2014, 40 p.

FOLEY, James \& RAMAND, Pete. Yes. The Radical Case for Scottish Independence. Londres : Pluto Press, 2014, 139 p.

HANHAM, H. J. Scottish Nationalism. Londres : Faber \& Faber, 1969, 250 p.

HASSAN, Gerry. Caledonian Dreaming. The Quest for a Different Scotland. Édimbourg : Luath Press, 2014, $252 \mathrm{p}$.

HM GOVERNMENT. Scotland Analysis: Currency and Monetary Policy. Londres : Stationery Office, Cm 8594, avril 2013, 118 p.

HM GOVERNMENT. UK Debt and the Scottish Independence Referendum. Londres : Stationery Office, janvier 2014, 5 p.

HM GOVERNMENT. Scotland Analysis: Assessment of a Sterling Currency Union. Londres : Stationery Office, $\mathrm{Cm} 8815$, février 2014, $60 \mathrm{p}$.

HM TREASURY. The Treasury and the Union. Londres, 21 janvier 2015, $13 \mathrm{p}$.

HOUSE OF COMMONS, BUSINESS, INNOVATION AND SKILLS COMMITTEE. The Implications of Scottish Independence on Business; Higher Education and Research; and Postal Services. Londres : Stationery Office, HC 504, août 2014, 30 p.

HOUSE OF COMMONS, SCOTTISH AFFAIRS COMMITTEE. The Referendum on Separation for Scotland: No Doubt-No Currency Union. Londres : Stationery Office, HC 499, juillet 2014, 46 p.

KAY, John. "Currency and Monetary Policy Options for an Independent Scotland", pp. 105-118 in Andrew GOUDIE (ed.), Scotland's Future. The Economics of Constitutional Change, Dundee: Dundee University Press, 2013.

KAY, John. “The Size of States-An Economic Analysis”, pp. 55-63 in Gerry HASSAN \& James MITCHELL (eds.), After Independence, Édimbourg : Luath Press, 2013.

KEATING, Michael \& HARVEY, Malcolm. Small Nations in a Big World. What Scotland Can Learn. Glasgow : Luath Press, 2014, 152 p.

MACASKILL, Kenny. Building a Nation. Édimbourg : Luath Press, 2004, 80 p.

MACDONALD, Ronald. "Scotland's Economic Future Post 2014", pp. 1-4 in SCOTTISH

PARLIAMENT, ECONOMY, ENERGY AND TOURISM COMMITTEE, Official Report, mars 2014.

MACLEOD, Dennis \& RUSSELL, Mike. Grasping the Thistle. Argyll : Glendaruel, 2006, 256 p.

MACWHIRTER, Iain. Disunited Kingdom. How Westminster Won a Referendum but Lost Scotland. Glasgow : Cargo, 2014, 174 p. 
MCCRONE, Gavin. Scottish Independence. Weighing up the Economics. Édimbourg : Birlinn, 2013, 160 p.

MAXWELL, Stephen. Arguing for Independence. Evidence, Risk and the Wicked Issues. Édimbourg : Luath Press, 2012, 181 p.

MURKENS, Jo Eric, JONES, Peter \& KEATING, Michael. Scottish Independence. A Practical Guide. Édimbourg : Edinburgh University Press, 2002, 318 p.

SCOTTISH GOVERNMENT. Scotland's Future. Your Guide to an Independent Scotland. Édimbourg, novembre 2013, 657p.

SCOTTISH GOVERNMENT. Outlook for Scotland's Public Finances and the Opportunities of Independence. Édimbourg, mai 2014, 48 p.

SCOTTISH GREEN PARTY. Green Yes. Édimbourg, 2013, 8p.

SCOTTISH SOCIALIST PARTY. Our Policies. Glasgow, 2014.

SCOTTISH TRADES UNION CONGRESS. A Just Scotland. Glasgow, février 2014, 60 p.

SILLARS, Jim. In Place of Fear II. Glasgow : Vagabond Voices, 2014, 94 p.

SIMPSON, David. “Independence: the Economic Issues”, pp. 121-131 in Neil MACCORMICK (ed.), The Scottish Debate. Essays on Scottish Nationalism, Londres : Oxford University Press, 1970.

SNP. SNP and You. Édimbourg, 1968, 20 p.

SNP. Independence in Europe. Make it Happen Now!. Édimbourg, 1992, 18 p.

SNP. Recovery in Scotland. Make it Happen Now!. Édimbourg, 1992, 14 p.

SNP. Horizons without Bars. Édimbourg, 1995, 64 p.

SNP. We Stand for Scotland. Édimbourg, 2001, 13 p.

SNP. Vote for Scotland. Édimbourg, 2004, 36 p.

SNP. We've Got what It Takes. Édimbourg, 2009, 20 p.

SNP. Elect a Local Champion. Édimbourg, 2010, 31 p.

THOMSON, Bob. “On Not Standing Still”, pp. 112-117 in James MAXWELL \& Owen D. EDWARDS (eds.), Why Not. Scotland, Labour and Independence. Édimbourg : Luath Press, 2014.

TORRANCE, David. 100 Days of Hope and Fear. How Scotland's Independence Referendum was Lost and Won. Glasgow : Luath Press, 2014, 188 p.

WATSON, Dave. “Challenging Neo-Liberal Orthodoxies”, pp. 183-190 in Pauline BRYAN \& Tommy KANE (eds.), Class, Nation and Socialism, The Red Paper on Scotland 2014, Glasgow : Glasgow Caledonian University Archives, 2013.

\section{NOTES}

1. Outre les fonctions d'unité de compte et de moyen d'échange, qui relèvent de la vie quotidienne, ils se sont familiarisés avec la fonction de réserve.

2. Comme l'a rappelé l'économiste John Kay, aucun État (à l'exception, peut-être, des États-Unis) ne peut être indépendant au plan économique dans un monde globalisé. John KAY, "The Size of States-An Economic Analysis", p. 62 in Gerry HASSAN \& James MITCHELL (eds.), After Independence, Édimbourg : Luath Press, 2013. 
3. Trois banques commerciales écossaises (Royal Bank of Scotland, Bank of Scotland et Clydesdale) ont conservé le droit de battre monnaie, en l'occurrence des billets de banque écossais, bénéficiant de la même valeur que les livres "sterling" et des mêmes garanties auprès de la Banque d'Angleterre. Ce privilège historique n'est pas cessible.

4. SNP, Statement of Aim and Policy, p. 227 in H. J. HANHAM, Scottish Nationalism, Londres : Faber \& Faber, 1969 ; SNP, The SNP and You, Édimbourg, 1968, p. 9 ; David SIMPSON, "Independence: the Economic issues", p. 127 in Neil MACCORMICK (ed.), The Scottish Debate: Essays on Scottish Nationalism, Londres : Oxford University Press, 1970. Neil MacCormick était le fils du fondateur du SNP.

5. SNP, Recovery in Scotland, Édimbourg, 1992, p. 2 ; SNP, We Stand for Scotland, Édimbourg, 2001, p.

7. Kenny MacAskill, député SNP au Parlement écossais, a admis que cette position servait les intérêts de l'Écosse et de son parti, in Building a Nation, Édimbourg : Luath Press, 2004, pp. 64-67.

6. Le Trésor avait imaginé cinq tests, relatifs aux conséquences de l'adoption de l'euro pour l'économie britannique. Seul celui concernant la City avait engendré des résultats positifs. Le rejet de l'euro semblait ainsi reposer sur des critères économiques alors qu'il résultait de pressions politiques, exercées par l'opinion publique comme par les Conservateurs.

7. SNP, Elect a Local Champion, Édimbourg, 2010, p. 22.

8. FISCAL COMMISSION WORKING GROUP, First Report, Édimbourg : Scottish Government, février $2013,7.38$ et 8.54 .

9. Ces participations représentaient $84 \%$ du capital de RBS et $42 \%$ de celui du groupe Lloyds qui avait dû intégrer HBoS, l'entité issue de la fusion entre Halifax et BoS.

10. Robbie DINWOODIE \& Michael SETTLE, “Chancellor Goes for Broke”, Herald, 14/02/14, p. 7.

11. Si la gauche écossaise s'accordait sur cette finalité, elle se divisait quant aux moyens, certains continuant à penser qu'il fallait maintenir la solidarité au plan britannique. Dave WATSON,“ Challenging Neo-Liberal Orthodoxies”, pp. 183-190 in Pauline BRYAN \& Tommy KANE (eds.), Class, Nation and Socialism, The Red Paper on Scotland 2014, Glasgow: Glasgow Caledonian University Archives, 2013.

12. Dans son Livre blanc de novembre 2013, le Gouvernement écossais a concédé que le choix appartiendrait aux dirigeants du nouvel État. SCOTTISH GOVERNMENT, Scotland's Future. Your Guide to an Independent Scotland, Édimbourg, novembre 2013, p.111.

13. Michael KEATING \& Malcolm HARVEY, Small Nations in a Big World. What Scotland Can Learn, Glasgow : Luath Press, 2014, chapitre 6.

14. SCOTTISH GOVERNMENT, 2013, op. cit., pp. 110-117.

15. HM GOVERNMENT, Scotland Analysis: Currency and Monetary Policy, Londres : Stationery Office, Cm 8594, avril 2013, pp. 7-8.

16. Ces estimations circulaient parmi les experts. Angus ARMSTRONG, \& Monique EBELL, Is this Plan B?, Londres : National Institute of Economic and Social Research, septembre 2014, p.4.

17. HM GOVERNMENT, UK Debt and the Scottish Independence Referendum, Londres: Stationery Office, 2014, 1.1.

18. SCOTTISH GOVERNMENT, Outlook for Scotland's Public Finances and the Opportunities of Independence, Édimbourg, mai 2014, 1.11.

19. Depuis la fin de sa convertibilité en or, en 1931, la livre était étroitement attachée à la réputation du Royaume-Uni, lequel n'avait jamais fait défaut dans le remboursement de ses dettes depuis le XVII ${ }^{\mathrm{e}}$ siècle. Angus ARMSTRONG \& Monique EBELL, Is this Plan B?, Londres: National Institute of Economic and Social Research, septembre 2014, p.2.

20. Mark CARNEY, The Economics of Currency Unions, Édimbourg: Scottish Council for Development and Industry, 2014, p. 14.

21. HM GOVERNMENT, Scotland Analysis: Assessment of a Sterling Currency Union, Londres : Stationery Office, Cm 8815, février 2014, pp. 7-8. 
22. Des économistes avaient démontré que la garantie d'une aide pouvait même stimuler la prise de risques excessifs. Angus ARMSTRONG \& Monique EBELL, "Commentary: Monetary Unions and Fiscal Constraints", National Institute Economic Review, n²28, mai 2014, F6.

23. C'était notamment l'analyse de l'économiste Ronald MACDONALD, "Scotland's Economic Future Post 2014", p. 3 in SCOTTISH PARLIAMENT, ECONOMY, ENERGY AND TOURISM COMMITTEE, Official Report, mars 2014. D'autres économistes avaient suggéré la vente des droits d'exploitation sur les hydrocarbures pour éponger la dette initiale. Angus ARMSTRONG \& Monique EBELL, Scotland's Currency Options, Londres : National Institute of Economic and Social Research, octobre 2013, p. 37.

24. Mark BROWN, “An Independent Scotland Would Have Neo-Colonial Ties with UK”, Guardian, 15/08/14. Le terme, destiné à dramatiser les enjeux, contrastait avec son ouvrage, paru quelques mois auparavant, visant à justifier le maintien de l'Écosse au sein du Royaume-Uni. Gordon BROWN, My Scotland, our Britain, Londres : Simon \& Schuster, 2014, p. 329.

25. FISCAL COMMISSION WORKING GROUP, The Economic Foundations of an Independent Scotland, Édimbourg : Scottish Government, août 2014, p. 10.

26. HOUSE OF COMMONS, SCOTTISH AFFAIRS COMMITTEE, The Referendum on Separation for Scotland: No Doubt-No Currency Union, Londres : Stationery Office, HC 499, juillet 2014, p. 3.

27. Voir, par exemple, l'avis du responsable de la campagne travailliste appelant à voter pour l'indépendance (Labour for Independence), Bob THOMSON, “On Not Standing Still”, p. 112 in James MAXWELL \& Owen D. EDWARDS (eds.), Why Not. Scotland, Labour and Independence, Édimbourg : Luath Press, 2014.

28. Cette autonomie avait été accordée par le Gouvernement de Tony Blair. Jim SILLARS, In Place of Fear II, Glasgow: Vagabond Voices, 2014, p. 11 ; SCOTTISH GREEN PARTY, For a Just and Welcoming Scotland, Édimbourg, mai 2014, p. 6.

29. Le rapport était de un à douze en 1707. En 2014, la dévaluation n'était plus à l'ordre du jour alors qu'elle était préconisée, dès l'accession de l'Écosse à l'indépendance, dans un ouvrage de 2006, co-signé par Mike Russell, devenu depuis ministre du Gouvernement écossais. Dennis MACLEOD \& Mike RUSSELL, Grasping the Thistle, Argyll : Glendaruel, 2006, 256 p.

30. Seul David Cameron était détesté par une plus forte proportion de sondés selon le sondage TNS-BMRB pour le supplément "Scotland Decides", Herald, 18 décembre 2013, p. 16.

31. Une commission économique de la Chambre des Communes, composée uniquement de députés unionistes, a même laissé entendre que l'UE pourrait s'opposer à l'union monétaire prônée par le SNP. HOUSE OF COMMONS, BUSINESS, INNOVATION AND SKILLS COMMITTEE, The Implications of Scottish Independence on Business; Higher Education and Research; and Postal Services, Londres : Stationery Office, HC 504, août 2014, paragraphe 27.

32. CBI, The Scottish Government's Plans for Independence, Glasgow, 2014, pp. 15-16.

33. Propos cités par le Herald, 26/08/14 (encart publicitaire) et 12/09/14, p. 6.

34. Ils affirmaient ne plus être en mesure de réaliser d'économies d'échelles pour transporter les marchandises vers un territoire périphérique. Marion DAKERS, "Retailers Warn Independence Would Push up Prices", Herald, 12/09/14, p. 9.

35. Kate DEVLIN, "Salmond: I Would Reach out to Rivals after Poll Victory", Herald, 16/09/14, p.7. 36. Edwige CAMP, «Les Pressions des milieux économiques et financiers sur le processus de dévolution en Écosse », Babel, n 17, 2008, p. 225.

37. ICM avait interrogé les Écossais pour The Scotsman, à trois reprises: la proportion de personnes soutenant la position du SNP était passée de $63 \%$ en février 2014 à $57 \%$ en septembre.cf www.whatscotlandthinks.org.

38. Les proportions respectives étaient $60 \%$ et $22 \%$ à la fin août, Herald, 1/09/14, p. 6 .

39. The Times, 20/09/14, p. 9.

40. La plupart sont demeurés neutres, deux ont incité à opter pour l'indépendance. Cela contrastait avec leur participation active à la rédaction d'un projet de dévolution, au sein de la 
Convention constitutionnelle, aux côtés (notamment) des Travaillistes et des LibérauxDémocrates, dans les années quatre-vingt-dix.

41. STUC, A Just Scotland, Glasgow, février 2014, p. 22.

42. Magnus GARDHAM, "Yes Scotland's Chief: Salmond's Plan to Share Pound Hit Victory Chances", Herald, 7/11/14, p. 6.

43. Certains unionistes ont déploré ces fuites, tel Alan COCHRANE, Alex Salmond: My Part in his Downfall, Londres : Biteback, 2014, p. 244.

44. HM TREASURY, The Treasury and the Union, Londres, 2015, p. 12.

45. C'est l'analyse d'un journaliste qui a voté en faveur de l'indépendance, Iain MACWHIRTER, Disunited Kingdom. How Westminster Won a Referendum but Lost Scotland, Glasgow : Cargo, 2014, p. 49.

\section{RÉSUMÉS}

Pendant la campagne référendaire de 2014, le SNP proposait de continuer à faire usage de la livre sterling dans le cadre d'une union monétaire entre l'Écosse indépendante et le reste du Royaume-Uni. Cette question technique est devenue un enjeu lorsque George Osborne, le Chancelier de l'Échiquier, a catégoriquement refusé cette perspective. La monnaie s'est imposée comme un emblème du débat. Selon les unionistes, l'économie écossaise ne pourrait de ce fait jamais être indépendante et prospère, raison pour laquelle nombre d'indépendantistes plébiscitaient la création d'une devise. Mais le SNP affirmait que les Écossais pourraient légitimement conserver leur patrimoine, tout en menant leurs propres politiques économiques et sociales. Les incertitudes persistantes ont finalement incité les derniers électeurs indécis à privilégier le Royaume-Uni.

In the 2014 referendum campaign the SNP claimed that an independent Scotland would use sterling as part of a currency union with the rest of the UK. George Osborne's blunt refusal to negotiate such a union turned this technical question into an issue. The currency symbolized the main arguments of the debate as the unionists argued that Scotland's economy would thus never be fully independent nor prosperous, which is the reason why many Yes campaigners advocated a new currency. Yet according to the SNP the Scots were entitled to retain a common asset while leading their own economic and social policies. Lingering doubt eventually prompted the remaining undecided voters to favour the UK.

\section{INDEX}

Mots-clés : Écosse, indépendance, référendum, souveraineté, monnaie

Keywords : Scotland, independence, referendum, sovereignty, currency

\section{AUTEUR}

\section{EDWIGE CAMP-PIETRAIN}

Université de Valenciennes 